\title{
Schwannoma of the esophagus: A case report
}

\author{
DONGLEI LIU, YANG YANG, YU QI, KAI WU and SONG ZHAO
}

Department of Thoracic Surgery, First Affiliated Hospital of Zhengzhou University, Zhengzhou, Henan 450052, P.R. China

Received October 24, 2014; Accepted August 5, 2015

DOI: $10.3892 / 01.2015 .3659$

\begin{abstract}
Esophageal schwannoma is a rare tumor among the thoracic diseases. The current study reports the case of a 62-year-old who presented with a 1-month history of dysphasia. A computed tomography scan of the chest revealed a solid mediastinal mass with a maximum diameter of $8 \mathrm{~cm}$, with inhomogeneous enhancement following injection of contrast. The mass was compressing the trachea and esophagus. Subsequent to cisplatin plus fluorouracil infusion and embolization with a gelatin sponge, a standard right-sided posterolateral surgical procedure was performed in the right thorax, with tumor enucleation and mucosal repair. The diagnosis of esophageal schwannoma was confirmed by pathological examination. Microscopic examination revealed spindle cells, with the positive expression of S-100 protein and negative expression results for endothelial membrane antigen and desmin, confirming the diagnosis of esophageal schwannoma.
\end{abstract}

\section{Introduction}

The majority of esophageal benign tumors are leiomyomas, and schwannoma is rare (1). Esophageal schwannoma was first described by Chatelin and Fissore in 1967 (2). It is difficult to diagnose these tumors by endoscopy and imaging, and they are finally diagnosed by pathological examination. Surgical resection is the main treatment for esophageal schwannoma, usually through a thoracotomy or esophagogastrostomy. Following complete excision, the prognosis of schwannoma is generally good and recurrence is rare (3). The present study reports a case of esophageal schwannoma with inhomogeneous enhancement following injection of contrast, in which a partial esophagetomy was performed following embolization under digital subtraction angiography (DSA). The study also describes the clinical and pathological features of this unusual tumor.

Correspondence to: Dr Song Zhao, Department of Thoracic Surgery, First Affiliated Hospital of Zhengzhou University, 1 Jiansge East Road, Zhengzhou, Henan 450052, P.R. China

E-mail: zhaosong@zzu.edu.cn

Key words: esophageal, schwannoma

\section{Case report}

A 62-year-old female presented to the First Affiliated Hospital of Zhengzhou University (Zhengzhou, Henan, China) in April 2014 with a 1-month history of dysphasia. A routine medical examination and the family history were unremarkable. A computed tomography (CT) scan of the chest showed a solid mediastinal mass with a maximum diameter of $8 \mathrm{~cm}$, with inhomogeneous enhancement following injection of contrast. The mass was compressing the trachea and esophagus (Fig. 1A). A hypermetabolic appearance (maximum standardized uptake value, 4.8; Fig. 1B) was noted on positron emission tomography (PET). Esophagoscopy demonstrated a smooth elevated lesion, at a distance of 17-24 cm from the incisor teeth. Based on these data, a mesenchymal tumor such as an esophageal leiomyoma was suspected.

Next, DSA was performed and the main feeding artery of tumor was shown to be the right thyrocervical trunk; $60 \mathrm{mg}$ cisplatin plus $1.0 \mathrm{mg}$ fluorouracil infusion was administered and embolization with a gelatin sponge was performed. Following the embolization, the chest CT indicated that the tumor size had not changed significantly, and there was a certain degree of necrosis in the center of tumor, with no marked enhancement compared with previously (Fig. 1C). A standard right-sided posterolateral surgical procedure was performed through the fifth intercostal space. The tumor was solid and dumbbell-shaped, originating from the muscular layer of esophagus, with no evident gaps between the esophageal mucosa and the membranous region of the trachea. Once the esophageal schwannoma had been enucleated, the esophageal mucosa and the membranous region of the trachea were repaired with sutures. Methylene blue was injected through the stomach tube and revealed no leakages.

On gross examination, the esophageal tumor was revealed to be an irregular and tenacious mass of $\sim 9 \mathrm{~cm}$ at the greatest dimension (Fig. 2A). Microscopic examination demonstrated spindle cells (Fig. 2B), with positive S-100 protein expression and negative endothelial membrane antigen and desmin expression, thus confirming the diagnosis of an esophageal schwannoma. Approximately 3 weeks later, an upper gastrointestinal angiography revealed an esophageal mucosa disorder, but the contrast agent went through smoothly. The patient was gradually transited from a liquid diet to a normal diet and was free of tumor recurrence following surgery. 

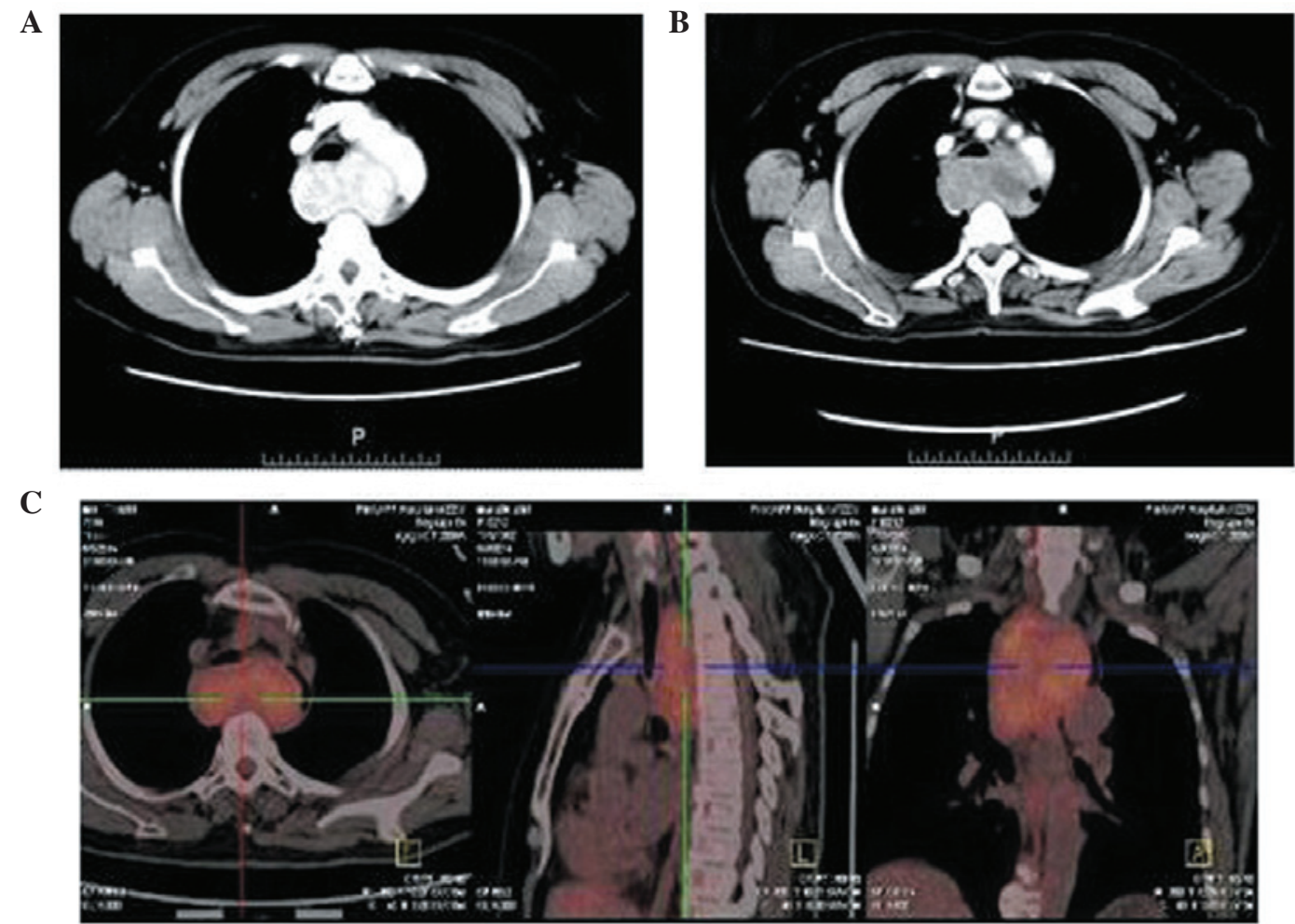

Figure 1. (A) Chest computed tomography demonstrating a large mediastinal mass compressing the trachea and esophagus, with inhomogeneous enhancement following injection of contrast agent and (B) with no marked enhancement following embolization under digital subtraction angiography. (C) Positron emission tomography showing a hypermetabolic appearance (maximum standardized uptake value, 4.8 ).
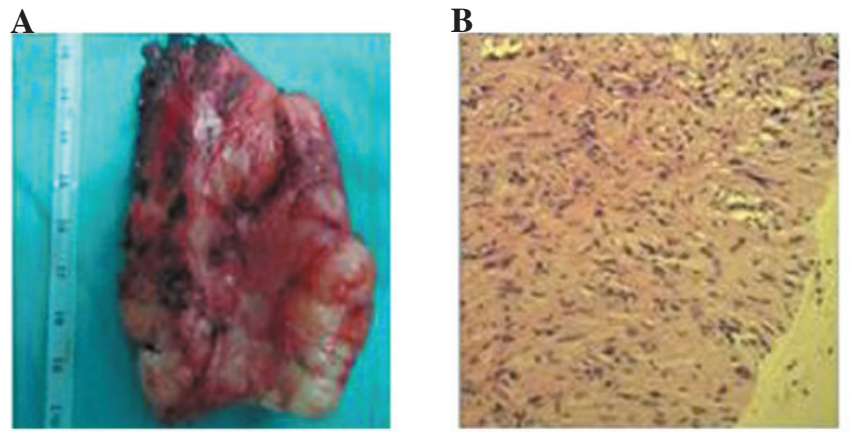

Figure 2. (A) Irregular and tenacious esophageal schwannoma. (B) Microscopic examination demonstrating spindle cells.

\section{Discussion}

The majority of esophageal tumors are malignant, and benign primary tumors of the esophagus are uncommon, representing a low percentage of all esophageal tumors (4). Esophageal schwannoma is a rare tumor, with the majority of literature cases originating in Asia. Reviews of previous case studies have recorded mediastinal masses with no marked reinforcement on CT enhancement scanning (5-7), this report indicated a benign esophageal schwannoma with inhomogeneous enhancement on CT scanning. Following the embolization of DSA, a thoracotomy with enucleation was performed to treat the schwannoma. Final diagnosis was made on histological and immunohistochemical basis: Esophageal schwannomas are typically characterized by palisading spindle cells histologically and tumor cells are immunohistochemically positive for S100, a characteristic marker of Schwann cells (8). The main treatment for esophageal schwannomas is surgical resection.

In summary, in the present study, a patient with a benign esophageal schwannoma was successfully treated via DSA and thoracotomy with enucleation. The final diagnosis of an esophageal schwannoma was made by pathological examination. The present case provides novel data suggesting that an esophageal schwannoma may also have a rich blood supply, which requires assessment via examinations prior to surgery.

\section{References}

1. Hsu SD, Cheng YL, Chen A and Lee SC: Esophageal schwannoma. J Formos Med Assoc 102: 346-348; 2003

2. Chatelin CL and Fissore A: Shwanome degenere de l'esophage. Confront Radio Anat Clin 7: 114, 1967.

3. Chen HC, Huang HJ, Wu CY, Lin TS and Fang HY: Esophageal schwannoma with tracheal compression. Thorac Cardiovasc Surg 54: 555-558, 2006

4. Kitada M, Matsuda Y, Hayashi S, Ishibashi K, Oikawa K and Miyokawa N: Esophageal schwannoma: A case report. World J Surg Oncol 11: 253, 2013

5. Liu T, Liu H, Yang C, Zhang X, Xu S and Liu B: Benign esophageal schwannoma compressing the trachea requiring esophagectomy: A case report. Thorac Cardiovasc Surg 61: 505-506, 2013.

6. Kassis ES, Bansal S, Perrino C, Walker JP, Hitchcock C, Ross P Jr, and Daniel VC: Giant asymptomatic primary esophageal schwannoma. Ann Thorac Surg 93: e81-e83, 2012.

7. Jeon HW, Kim KS, Hyun KY and Park JK: Enucleation of giant esophageal schwannoma of the upper thoracic esophagus: Reports of two cases. World J Surg Oncol 12: 39, 2014.

8. Ferrante M, Khan A, Fan C and Jelloul FZ: Schwannoma of the cervical esophagus. Rare Tumors 6: 5361, 2014. 